\title{
Residential Community Open-Up Strategy Based on Prim's Algorithm and Neural Network Algorithm
}

\author{
Ximing $\mathrm{Lv}^{1,2}$, Ang $\mathrm{Li}^{3}$, Shunkai Zhang ${ }^{3}$, Jianbao $\mathrm{Li}^{3}$ \\ ${ }^{1}$ School of Mathematical Sciences, Inner Mongolia University, Hohhot, China \\ ${ }^{2}$ School of Statistics and Mathematics, Inner Mongolia University of Finance and Economics, Hohhot, China \\ ${ }^{3}$ School of Finance, Inner Mongolia University of Finance and Economics, Hohhot, China \\ Email: lvximing@vip.163.com
}

How to cite this paper: Lv, X.M., Li, A., Zhang, S.K. and Li, J.B. (2017) Residential Community Open-Up Strategy Based on Prim's Algorithm and Neural Network Algorithm. Journal of Applied Mathematics and Physics, 5, 551-567.

https://doi.org/10.4236/jamp.2017.52047

Received: January 5, 2017

Accepted: February 25, 2017

Published: February 28, 2017

Copyright $\odot 2017$ by authors and Scientific Research Publishing Inc. This work is licensed under the Creative Commons Attribution International License (CC BY 4.0).

http://creativecommons.org/licenses/by/4.0/

\begin{abstract}
"Open community" has aroused widespread concern and research. This paper focuses on the system analysis research of the problem that based on statistics including the regression equation fitting function and mathematical theory, combined with the actual effect of camera measurement method, Prim's algorithm and neural network to "Open community" and the applicable conditions. Research results show that with the increasing number of roads within the district, the benefit time gradually increased, but each type of district capacity is different.
\end{abstract}

\section{Keywords}

Open Community, Regression Analysis, Prim's Algorithm, Graph Theory, Neural Net-Work Algorithm

\section{Introduction}

Capillaries are reticular throughout the body, connecting arteries and veins. If the capillary is clogged, it will affect the blood flow and material exchange between arteries and veins. For urban traffic, "capillaries" are small roads that people are not interested in. If the "capillaries" blocked, then the "artery" and "vein" are no longer efficient [1]. How to solve the problem of traffic congestion by reasonable "open community" to improve the density of the road network is the focus of our study.

In order to study this huge problem, we decided to establish a mathematical model, and then empirical analysis. This requires us to establish a suitable index system, to evaluate the "open community" to control the role of road congestion. 
After analysis, we established the following index evaluation system (Figure 1).

Road capacity, we can use a certain vehicle through a certain area of the minimum time to measure. The term "the benefit time" is defined as the time difference between a roadway around a residential area that permits traffic and does not allow traffic. The "the benefit time" is determined by the number of lanes and the speed, and the speed is related to the traffic density

\section{Data Acquisition and Analysis}

\subsection{Regional Analysis and Selection}

As of September 11, 2016, there were 23 provinces, 4 municipalities, 2 special administrative regions and 5 autonomous regions in China. A total of 660 one, two, three-tier cities, each city car capacity and community structure is different, in order to study the problem in a better environment, select a suitable city is essential [2].

\subsubsection{The Selection of Hohhot Region}

Region selection reason:

1) Motor vehicle ownership is more appropriate, the majority of private cars, Hohhot, China can represent most of the city level, and there is great room for optimization.

2) This city is located in the plateau area. Roads are mostly straight; car congestion is almost free from geographical factors.

3) Residential area characterized as "Large mixed, small settlements", forming a number of residential circle, conducive to the investigation and research.

Based on the above reasons, the selected research city is Hohhot, and the selected community is the representative district in this city.

\subsubsection{The Selection of Residential Areas}

According to the data obtained from the traffic management department of Hohhot, as shown in the blue area for most of the private car owner residential area, the figures (Figure 2) shown are the percentage of private car ownership in the area. In our data, we selected the smallest percentage (red five-pointed star " $\star$ " mark) area and representative area, as our research area and field observation area. Traffic flow around it can be approximated according to the following formula:

Traffic flow around the residential area $=$ total traffic volume $\times$ The percentage.

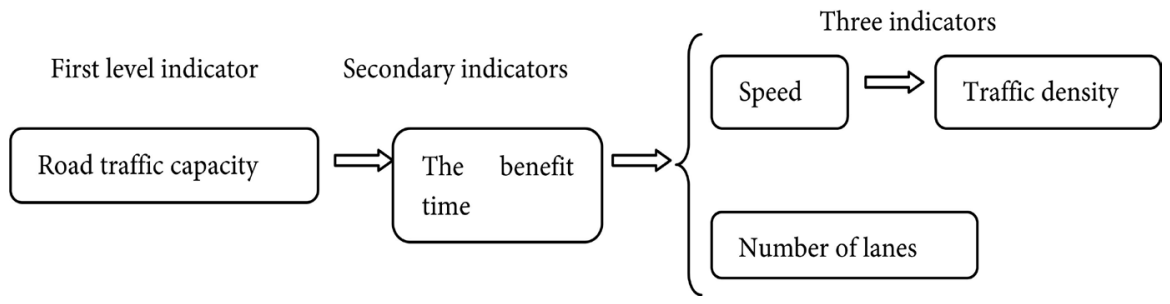

Figure 1 . The following index evaluation system. 


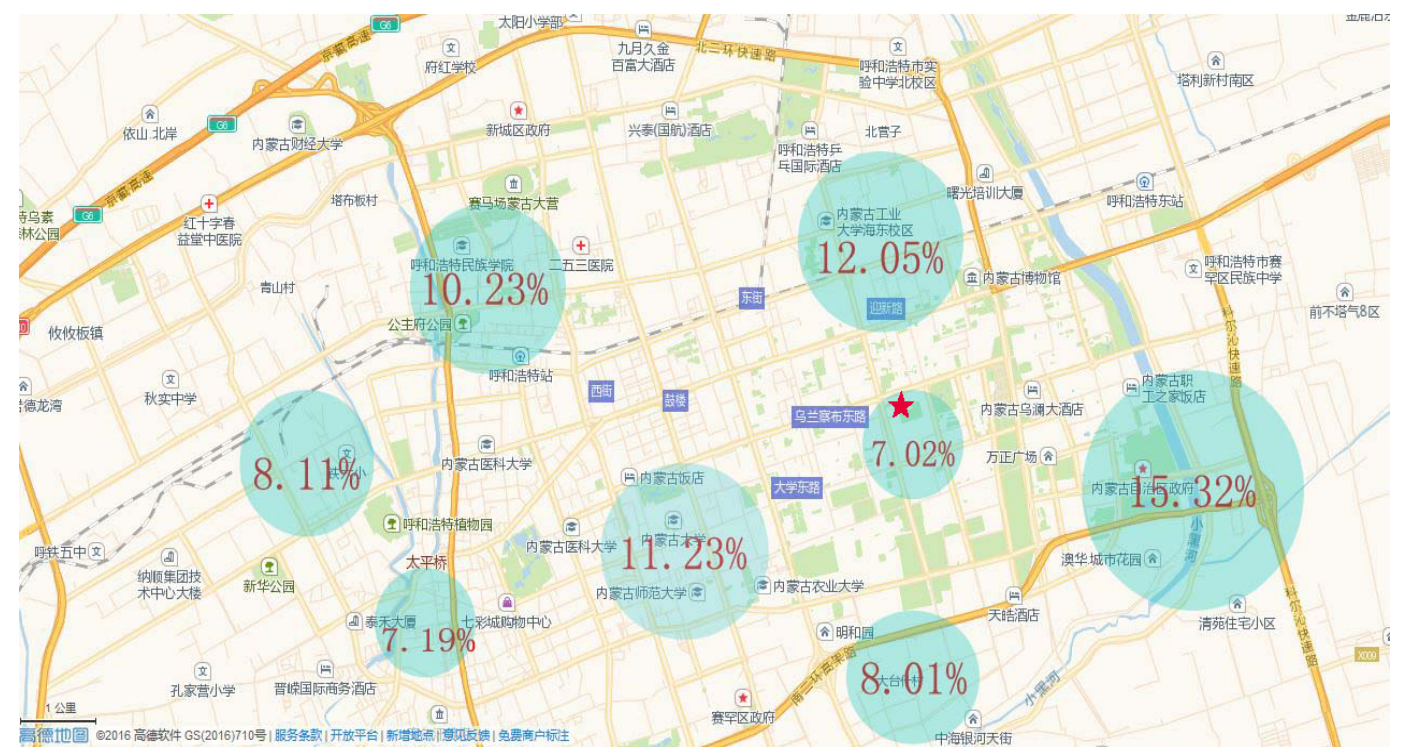

Figure 2. The map of Hohhot.

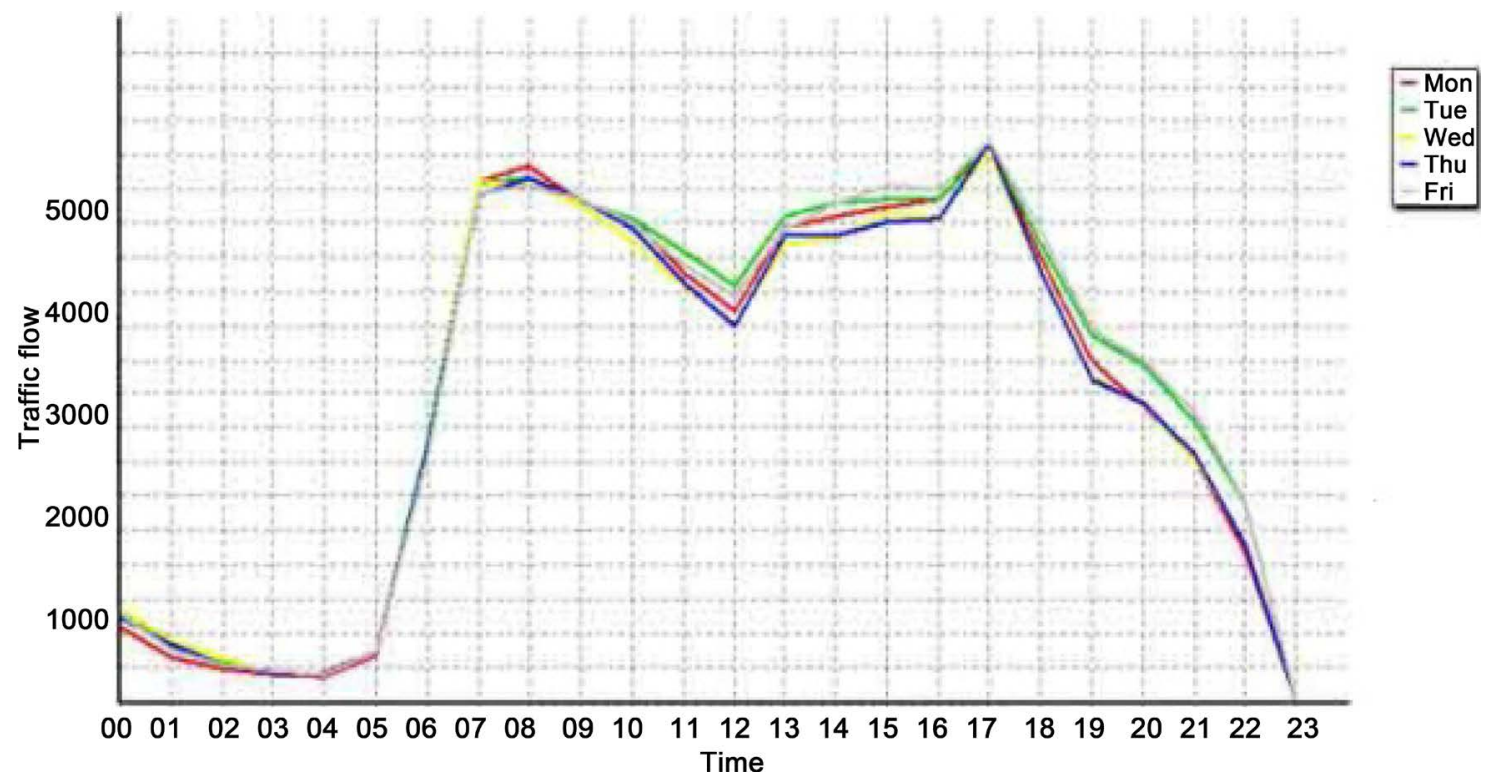

Figure 3. "Double hump".

\subsection{Acquisition of Traffic Flow Data}

\subsubsection{The Average Hourly Traffic Data Acquisition}

Through the "2011-2013 focus on environmental protection in key urban road traffic noise monitoring situation (2014)", we found that the average hourly traffic volume in Hohhot was 2231 Vehicle/hour [3].

\subsubsection{The Trend of the Daily Traffic Volume in Hohhot}

Through the study of many authoritative data, we find that the trend of traffic flow in many cities is "double hump [4]" (Figure 3).

After a lot of data query, we found that there is a function similar to the "double hump", through the modification and adjustment of this function, we get a "double-like hump" function (Figure 4). 


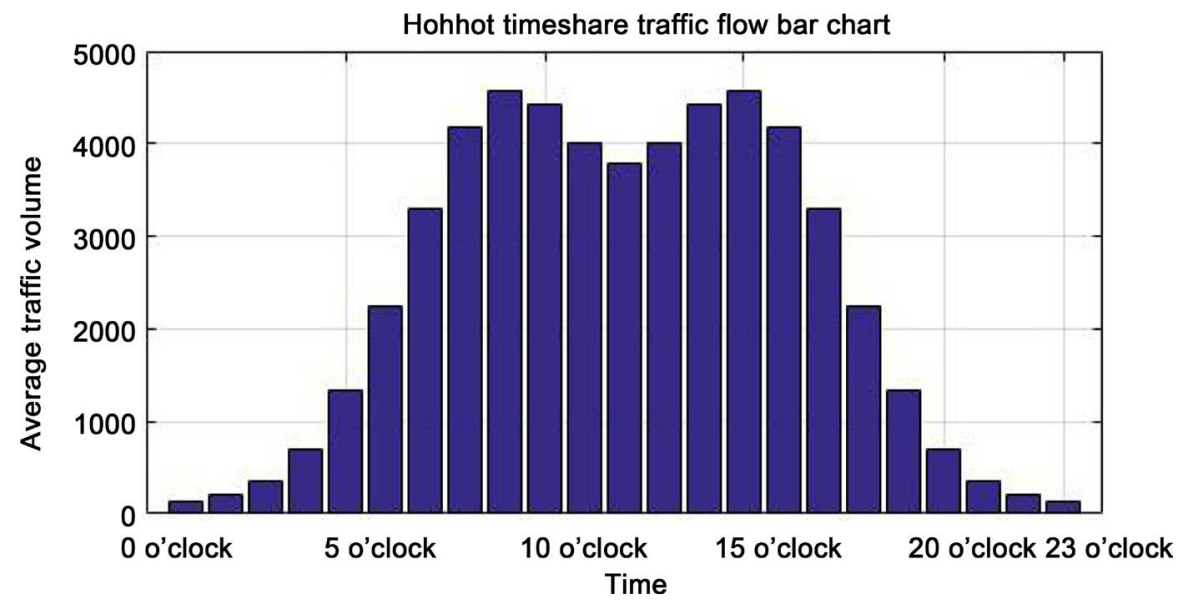

Figure 4. Hohhot timeshare flow bar chart.

$$
\mathrm{y}=\left(\left(\left(\frac{x}{4}-3\right)^{2}+0.5\right) \times \mathrm{e}^{-\left(\frac{x}{4}-3\right)^{2}+2}+0.1\right) \times 1000
$$

In the follow-up analysis, the total traffic volume of the whole city in different time periods will be calculated by this function.

\section{Preliminary Results}

Through the analysis of the problem, we choose an algorithm in graph theoryPrim's algorithm [5] [6] [7] to resolve this issue. It finds a subset of the edges that form a tree that includes every vertex, where the total weight of all the edges in the tree is minimized. The algorithm operates by building this tree one vertex at a time, from an arbitrary starting vertex, at each step adding the cheapest possible connection from the tree to another vertex. We believe that Prim's algorithm can be a good solution to this problem.

In general, area around the crossroads can be formed well shape(Figure 5).

Assume the gate is at points $A$ and $D$, so departure from $A_{1}$ and $A_{2}$ or $D_{1}$ and $D_{2}$ the departure traffic is the same. So this situation can be simplified into the following road network simulation map (Figure 6).

\subsubsection{Traffic Velocity-Density Relation Model}

In order to obtain the necessary data, we use the Traffic Engineering photogrammetry [8] [9] [10]. We were erected VCR in Wulanchabu East Road and the pedestrian bridge at University Road. Finally, select a number of private cars in the video, calculate the speed. Assuming constant speed, select one of the representative speed as the fitting data points in the speed value, in this selection, we assume that the vehicle travels at a constant speed and then uses the representative velocity as the velocity value in the fitted data points, Through the "double hump" function and the surrounding area traffic flow calculation formula, calculate the density of different time periods. A total of 24 groups were selected (Figure 7).

After obtaining the data, we are ready to use MATLAB software for regression 


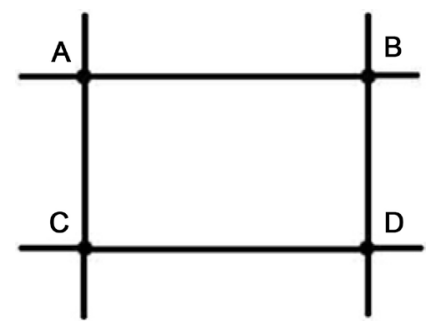

Figure 5. Well shape.

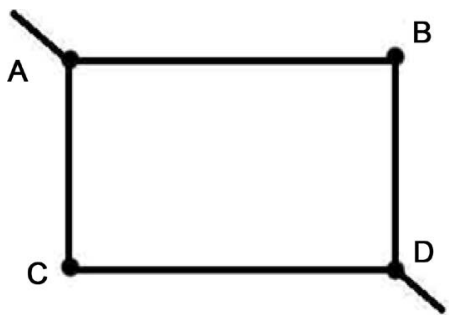

Figure 6. Road network simulation map.

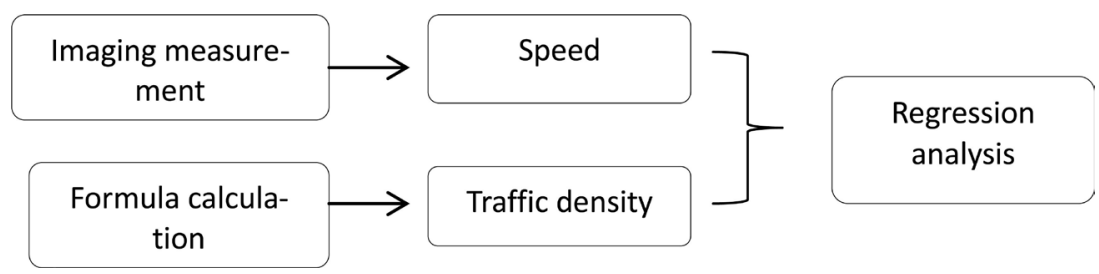

Figure 7. The analysis chart.

analysis [11] [12] [13] of the data to quantify the relationship between density and speed.

In order to quantitatively express the relationship between density and velocity, we then carry out different degrees of regression analysis in order to find the function expression between the two.

\subsubsection{Linear Regression Models}

Its linear regression model is shown in Figure 8.

\section{1) Square regression equation}

Its square regression model is shown Figure 9.

\section{2) Cubic regression equation}

Its cubic regression model is shown in Figure 10.

\section{3) Neural Network Optimal Regression}

We use MATLAB software for neural network Levenberg-Marquardt [14] [15] [16] operation. Set Training 70\%, Validation 15\%, Text 15\%, to obtain the optimal regression curve (Figure 11).

Remnant analysis of neural network and raining, Validation, Text of the correlation coefficient are shown in Figure 12 and Figure 13.

After several iterations, we find that the correlation coefficient is optimal when the high-order regression equation, but the correlation coefficient change from the second order is not particularly obvious, so we then use second-order regression equation as a function of speed and density relational expression. 


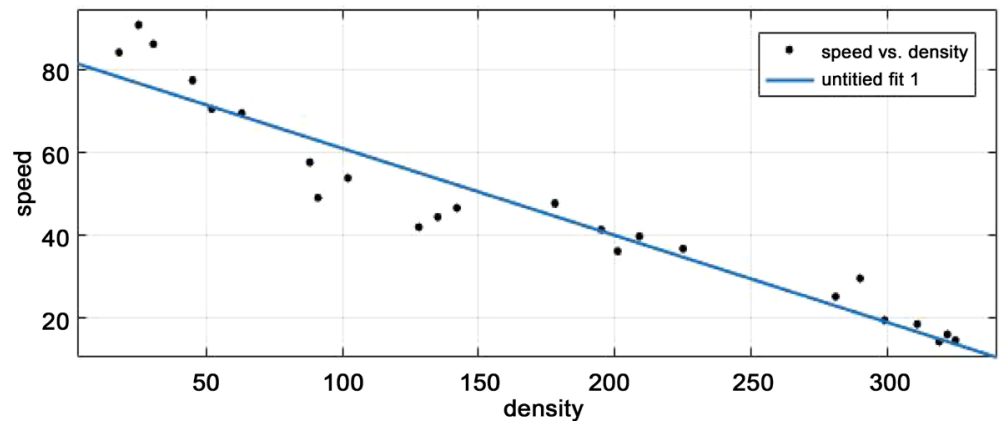

Figure 8. The linear regression curve.

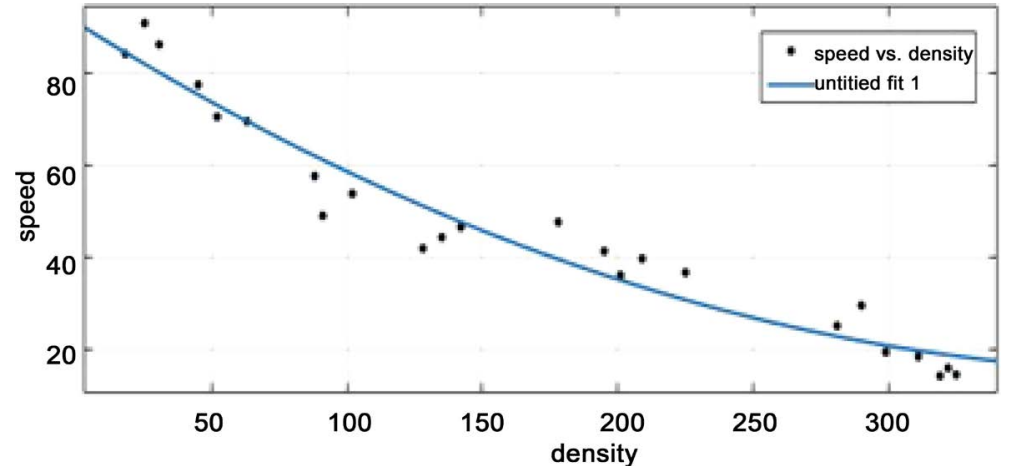

Figure 9. The square regression curve.

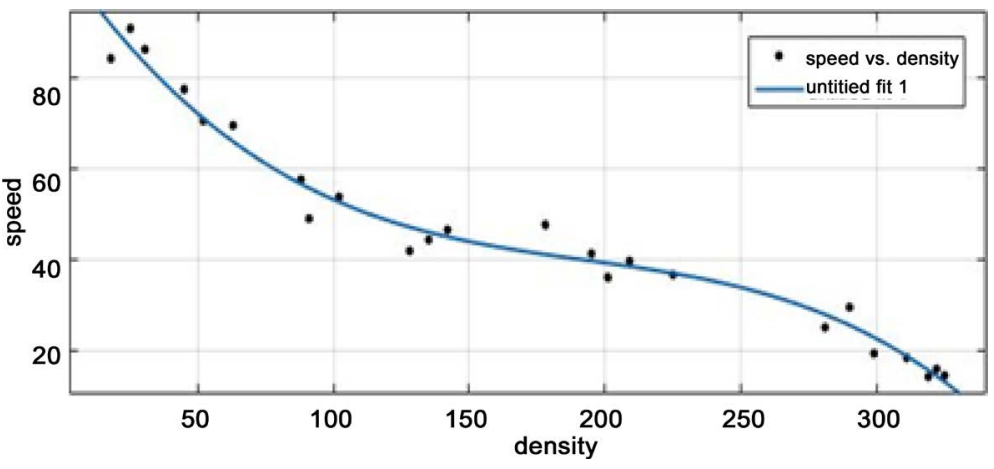

Figure 10. The cubic regression curve.

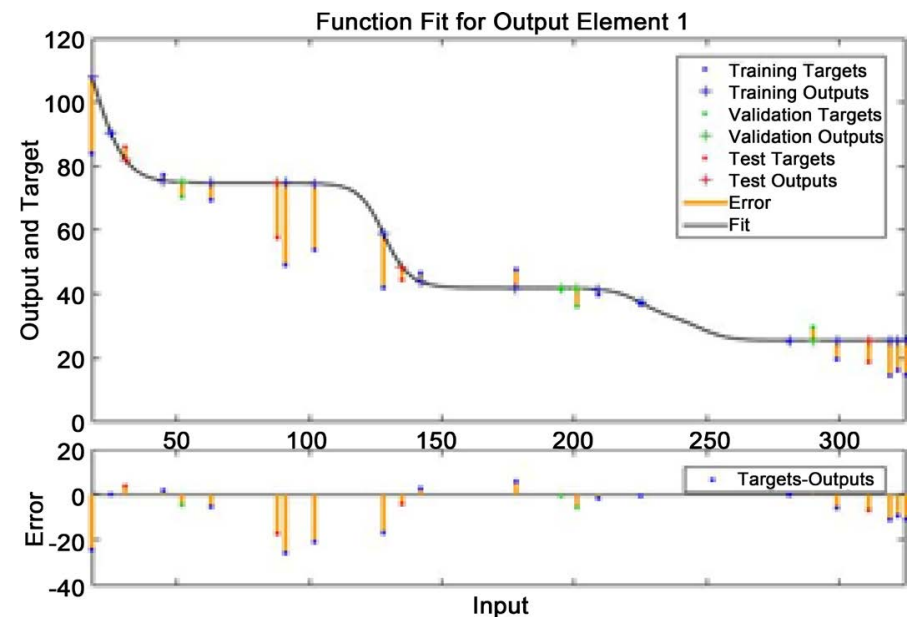

Figure 11. The neural network optimal regression curve. 


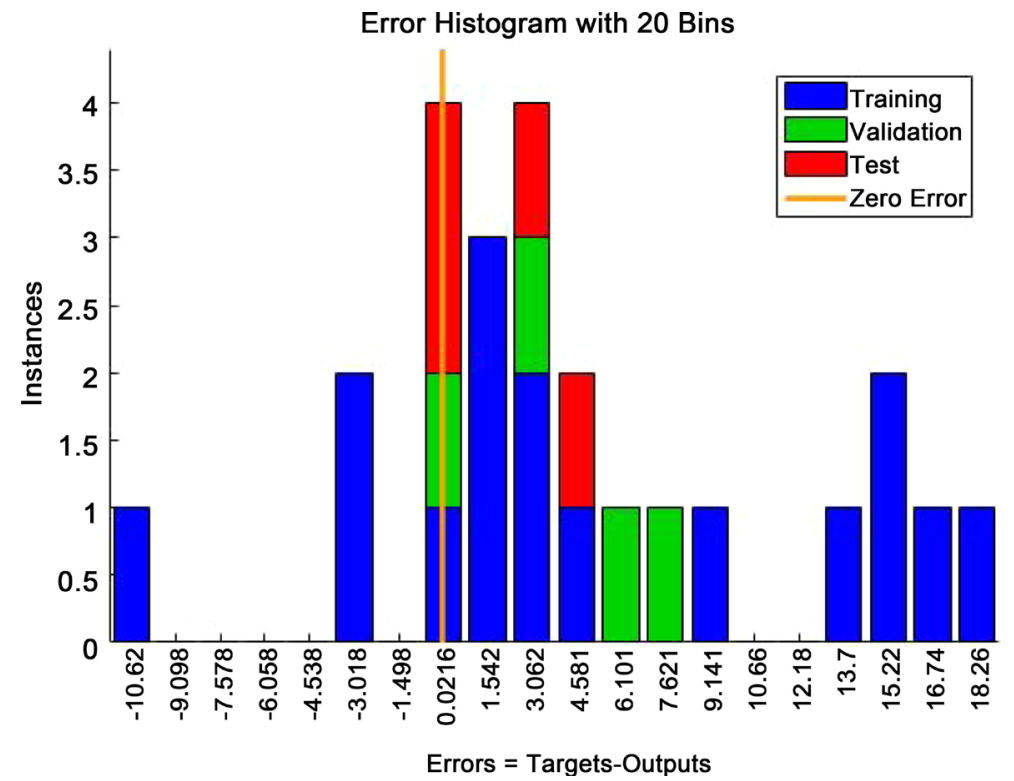

Figure 12. Residual analysis of neural network.
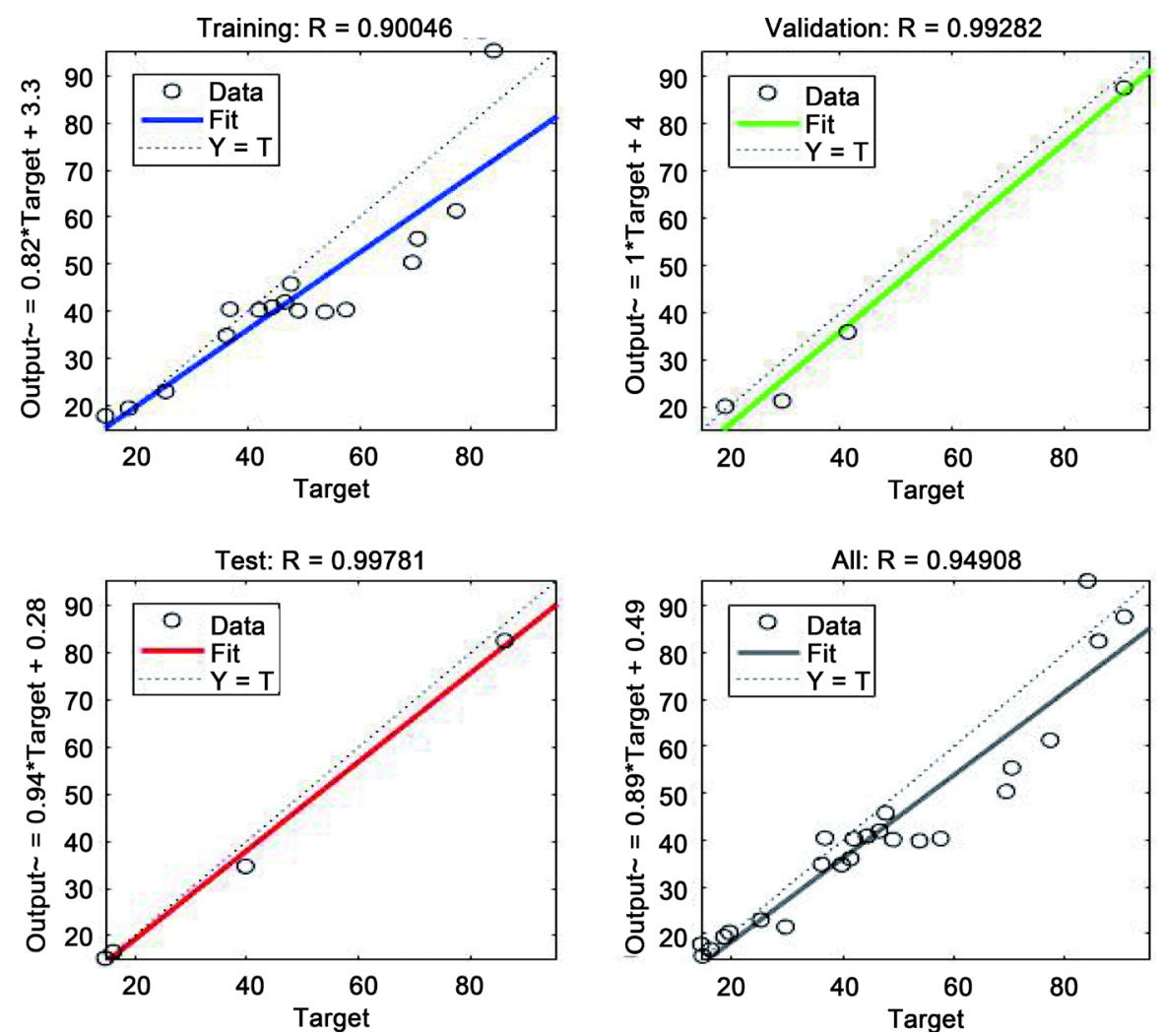

Figure 13. Correlation coefficient analysis.

\subsubsection{Put Forward the Mathematical Model}

According to the Prim's algorithm and permutation and combination method, we simulate the driver's route selection method:

1) In the node a wait, in front of the fork in the road $1,2, \ldots$, after selection into step 2.

2) After the observation, select the lowest traffic flow in the fork in the road, 
to reach the next node, go to step 3.

3) The node is the end of the loop term to stop, if not the end, continue with step 1 cycle.

In the steady state, with the formula for calculating the profit time to get this set of formulas:

$$
\Delta T=\sum_{i}\left(T_{1}^{i}-T_{2}^{i}\right)=\sum_{i}\left(T_{3}^{i}-T_{4}^{i}\right)=\sum_{i}\left(T_{5}^{i}-T_{6}^{i}\right), \quad(i=1,2,3 \cdots)
$$

In which, $T_{1}^{i}$ is the commuting time required before opening section $\mathrm{Yi}, T_{2}^{i}$ is the commuting time required after opening section $\mathrm{Yi}, T_{3}^{i}$ is the length of the before opening section $\mathrm{Yi} /$ the speed of the Vehicle on the road before opening section $\mathrm{Yi}, T_{4}^{i}$ is the length of the after opening section $\mathrm{Yi} /$ the speed of the vehicle on the road after opening section $\mathrm{Yi}, T_{5}^{i}$ is the length of the before opening section $\mathrm{Yi} /$ the traffic density of the before opening section $\mathrm{Yi}, T_{6}^{i}$ is the length of the after opening section $\mathrm{Y} i /$ the traffic density of the after opening section Yi.

\subsection{Simple Model Simulation}

\subsubsection{Single-Lane Community}

By simulating the simplest cell structure, we can obtain the following road network simulation map (Figure 14).

Let $A B$ segment be $n_{1}$ lane, $B D$ segment be $n_{2}$ lane, $C D$ is $n_{3}$ lane, $A C$ segment is $n_{4}$ lane.

According to the driver routing method, we can assume that in the case of stability, $A E$ and $A C$ road traffic density is the same. Let this traffic density be $\rho$, And traffic flow density caused by traffic flow in other directions is denoted by $\rho_{n}, \rho_{e}, \rho_{s}, \rho_{w}$. After opening the cell, the density of each section is

The ratio of traffic density between $A E$ and $A C$ links is $\quad 1: 1$

$$
A E=\rho, \quad A C=\rho
$$

When the vehicle arrives at point $E$, the traffic density of $E F$ and $E B$ sections is equal. The ratio of traffic density between $E B$ and $A E$ is $1:\left(1+n_{-} 1\right)$ :

$$
E B=\frac{\rho \times 1}{1+n_{1}}, E F=\frac{\rho \times 1}{1+n_{2}}
$$

When the vehicle arrives at point $B$, the traffic density of $E B$ and $B D$ is $n_{1}: n_{2}$ :

$$
B D=\frac{\rho \times n_{1}}{\left(1+n_{1}\right) \times n_{2}}
$$

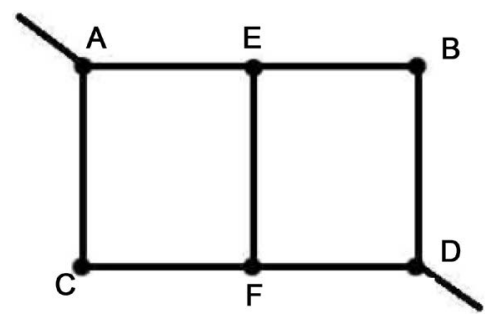

Figure 14. Single-lane community net. 
When the vehicle arrives at the point $F$, the traffic density of the $F D$ section comes from the traffic density of the $E F$ section and the $C F$ section:

$$
F D=\frac{\rho \times n_{4}+\frac{\rho}{1+n_{1}}}{n_{3}}
$$

When the vehicle arrives at point $C$, the ratio of the traffic flow density of $C F$ road segment to the traffic flow density of $A C$ link is $n_{3}: n_{4}$ :

$$
C F=\frac{\rho \times n_{4}}{n_{3}}
$$

Before the "Open community", the density (Figure 15) of each link is:

$$
A B=\rho, \quad A C=\rho, \quad B D=\frac{\rho \times n_{1}}{n_{2}}, \quad C D=\frac{\rho \times n_{4}}{n_{3}}
$$

Let $\Delta \mathrm{T}$ be the time gain after opening the cell:

$$
\begin{aligned}
\Delta T= & \left\{\frac{n_{1}}{n_{1}+1} \times\left[\frac{Y_{1}}{f\left(\rho+\rho_{n}\right)}-\frac{Y_{1}}{f\left(\frac{\rho_{1} \times 1}{1+n_{1}}+\rho_{n}\right)}+\frac{2 \times Y_{2}}{f\left(\frac{\rho \times n_{1}}{n_{2}}+\rho_{e}\right)}\right.\right. \\
& \left.-\frac{2 \times Y_{2}}{f\left(\frac{\rho \times n_{1}}{\left(1+n_{1}\right) \times n_{2}}+\rho_{e}\right)}\right]+\frac{1}{n_{1}+1} \times\left[\frac{2 \times Y_{2}}{f\left(\frac{\rho \times n_{1}}{n_{2}}+\rho_{e}\right)}-\frac{2 \times Y_{2}}{f\left(\frac{\rho \times 1}{1+n_{1}}\right)}\right. \\
& +\frac{Y_{1}}{f\left(\frac{\rho \times n_{4}}{n_{3}}+\rho_{s}\right)}-\frac{Y_{1}}{\left.\left.f\left(\frac{\rho \times n_{4}+\frac{\rho}{1+n_{1}}}{n_{3}}+\rho_{s}\right)\right]\right\}} \\
& +\frac{n_{4}}{n_{1}+n_{4}} \times\left[\frac{Y_{1}}{f\left(\frac{\rho \times n_{4}}{n_{3}}+\rho_{s}\right)}-\frac{f\left(\frac{\rho+n_{4}+\frac{\rho}{1+n_{1}}}{f}+\rho_{s}\right.}{n_{3}}\right]
\end{aligned}
$$

\subsubsection{T-Shaped Road District}

By modeling a slightly more complex cell structure, we can obtain the following plot road network simulation.

Let $A B$ segment be $n_{1}$ lane, $B D$ segment be $n_{2}$ lane, $C D$ is $n_{3}$ lane, $A C$ segment is $n_{4}$ lane (Figure 16).

The ratio of traffic density between $A E$ and $A C$ links is $\quad 1: 1$

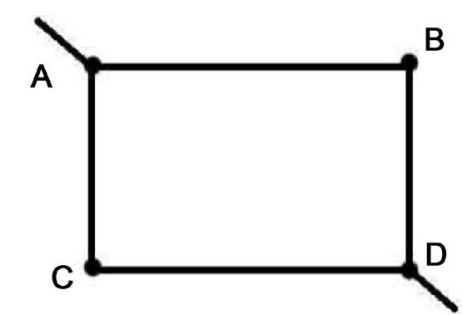

Figure 15. Open community net. 


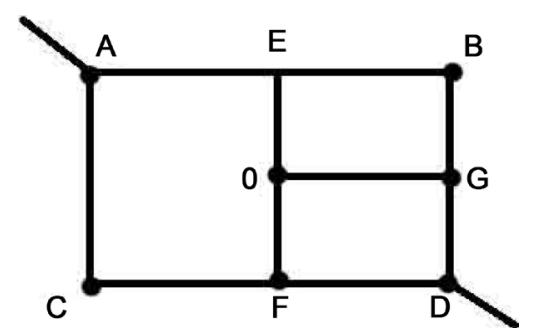

Figure 16. T-shaped road district.

$$
A E=\rho, A C=\rho
$$

When the vehicle arrives at point $E$, the traffic density of $E B$ and $E O$ sections is equal. The ratio of traffic density between $E B$ and $A E$ is $\left.1:\left(1+n_{1}\right)\right)$ :

$$
E B=\frac{\rho \times 1}{1+n_{1}}, E O=\frac{\rho}{1+n_{1}}
$$

When the vehicle arrives at point $B$, the traffic density of $E B$ and $B D$ is $n_{1}: n_{2}$ :

$$
B G=\frac{n_{1} \times \rho}{\left(1+n_{1}\right) \times n_{2}}
$$

The traffic density of the $C F$ link is determined by the $A C$ link:

$$
C F=\frac{\rho \times n_{4}}{n_{3}}
$$

When the vehicle arrives at point $O$, the traffic density of $E O$ is divided into $O G$ and $O F$ :

$$
O G=\frac{1 \times \rho}{2 \times n_{1}+2}, F O=\frac{1 \times \rho}{2 \times n_{1}+2}
$$

The traffic density of the $G D$ section is gathered by the traffic density of the $O G$ link and the $B G$ link:

$$
G D=\frac{\left[\frac{\rho \times n_{1}}{1+n_{1}}+\frac{1 \times \rho}{\left(2 \times n_{2}+2\right)}\right]}{n_{2}}
$$

$F G$ road traffic flow density from the $O F$ section and the $C F$ section of the traffic flow density from the pool, then:

$$
F D=\frac{\left[\frac{1 \times \rho}{2 \times n_{1}}+n_{4} \times \rho\right]}{n_{3}}
$$

Before the "Open community", the density (Figure 15) of each link is:

$$
\begin{aligned}
& A B=\rho, B D=\frac{n_{1} \times \rho}{n_{2}}, \\
& C D=\frac{\rho \times n_{4}}{n_{3}}, A C=\rho
\end{aligned}
$$

Let $\Delta T$ be the time gain after opening the cell: 


$$
\begin{aligned}
& \Delta T=\frac{n_{1}}{n_{1}+n_{4}} \times \frac{n_{1}}{n_{1}+1} \times\left[\frac{Y_{1}}{f\left(\frac{\rho}{1+n_{1}}+\rho_{n}\right)}-\frac{Y_{1}}{f\left(\frac{\rho}{1+n_{1}}+\rho_{n}\right)}+\frac{2 \times Y_{2}}{f\left(\frac{\rho \times n_{1}}{n_{2}}+\rho_{e}\right)}\right. \\
& \left.-\frac{Y_{2}}{f\left(\frac{\rho \times n_{1}}{\left(1+n_{1}\right) \times n_{2}}+\rho_{e}\right)}-\frac{Y_{2}}{f\left(\frac{\left[\frac{1 \times \rho}{2 \times n_{1}}+n_{4} \times \rho\right]}{n_{3}}+\rho_{e}\right)}\right] \\
& +\frac{n_{1}}{n_{1}+1} \times \frac{1}{2 \times n_{1}+2} \times\left[\frac{Y_{1}}{f\left(\rho+\rho_{n}\right)}+\frac{2 \times Y_{2}}{f\left(\frac{n_{1} \times \rho}{n_{2}}+\rho_{e}\right)}-\frac{Y_{2}}{f\left(\frac{\rho}{1+n_{1}}\right)}\right. \\
& \left.-\frac{Y_{2}}{f\left(\frac{1 \times \rho}{2 \times n_{1}+2}\right)}-\frac{Y_{1}}{f\left(\frac{\left[\frac{1 \times \rho}{2 \times n_{1}}+n_{4} \times \rho\right]}{n_{3}}\right)}\right] \\
& +\frac{n_{1}}{n_{1}+n_{4}} \times \frac{1}{2 \times n_{1}+2} \times\left[\frac{Y_{1}}{f\left(\rho+\rho_{n}\right)}+\frac{2 \times Y_{2}}{f\left(\frac{n_{1} \times \rho}{n_{2}}+\rho_{e}\right)}\right. \\
& \left.-\frac{Y_{2}}{f\left(\frac{\rho}{1+n_{1}}\right)}-\frac{Y_{2}}{f\left(\frac{1 \times \rho}{2 \times n_{1}+2}\right)}-\frac{Y_{2}}{\left.f\left(\frac{\left[\frac{\rho \times n_{1}}{1+n_{1}}+\frac{1 \times \rho}{2 \times n_{2}+2}\right]}{n_{2}}\right]\right)}\right] \\
& +\frac{n_{1}}{n_{1}+n_{4}} \times\left[\frac{Y_{1}}{f\left(\frac{\rho \times n_{4}}{n_{3}}+\rho_{s}\right)}-\frac{Y_{1}}{f\left(\frac{\left.\frac{1 \times \rho}{2 \times n_{1}}+n_{4} \times \rho\right)}{n_{3}}\right)+\rho_{s}}\right]
\end{aligned}
$$

Through the empirical analysis of the study, we come to the following conclusions as Table 1.

Next, the evaluation system is used to test the traffic flow of the residential vehicles: the traffic volume density and the cell type are regulated under the premise of controlling the number of lanes around the cells.

After selecting the mean (175) and maximum (325) in the series data, we found that the same time, the yield time with the number of lanes in the district continues to lengthen. When only the density changes, the experiment chooses the peak data 325 , the single-lane cell and the $T$-shaped road plot gain time is negative, which reflects the two communities on the morning and evening peak

Table 1. The benefit time of a single lane.

\begin{tabular}{ccc}
\hline \multicolumn{2}{c}{ Data Sheet } \\
\hline Traffic density & The benefit time of a single lane & T-section of the benefits of time \\
\hline 170 & 1.9222 & 2.6883 \\
325 & -50.33305 & -17.1311 \\
\hline
\end{tabular}


traffic capacity is limited. Community open only play a role in slowing traffic congestion, rather than cure.

\section{Further Study}

\subsection{Actual Cell Simulation}

\subsubsection{Cross Road Community}

This area is a selected area of the old district and the structure is relatively simple. The main road is a one-way street and the main road network structure marked in the map. The area around the road is a two-way street. Now we make an analysis of a problem about whether the area is conducive to ease the surrounding road congestion (Figure 17).

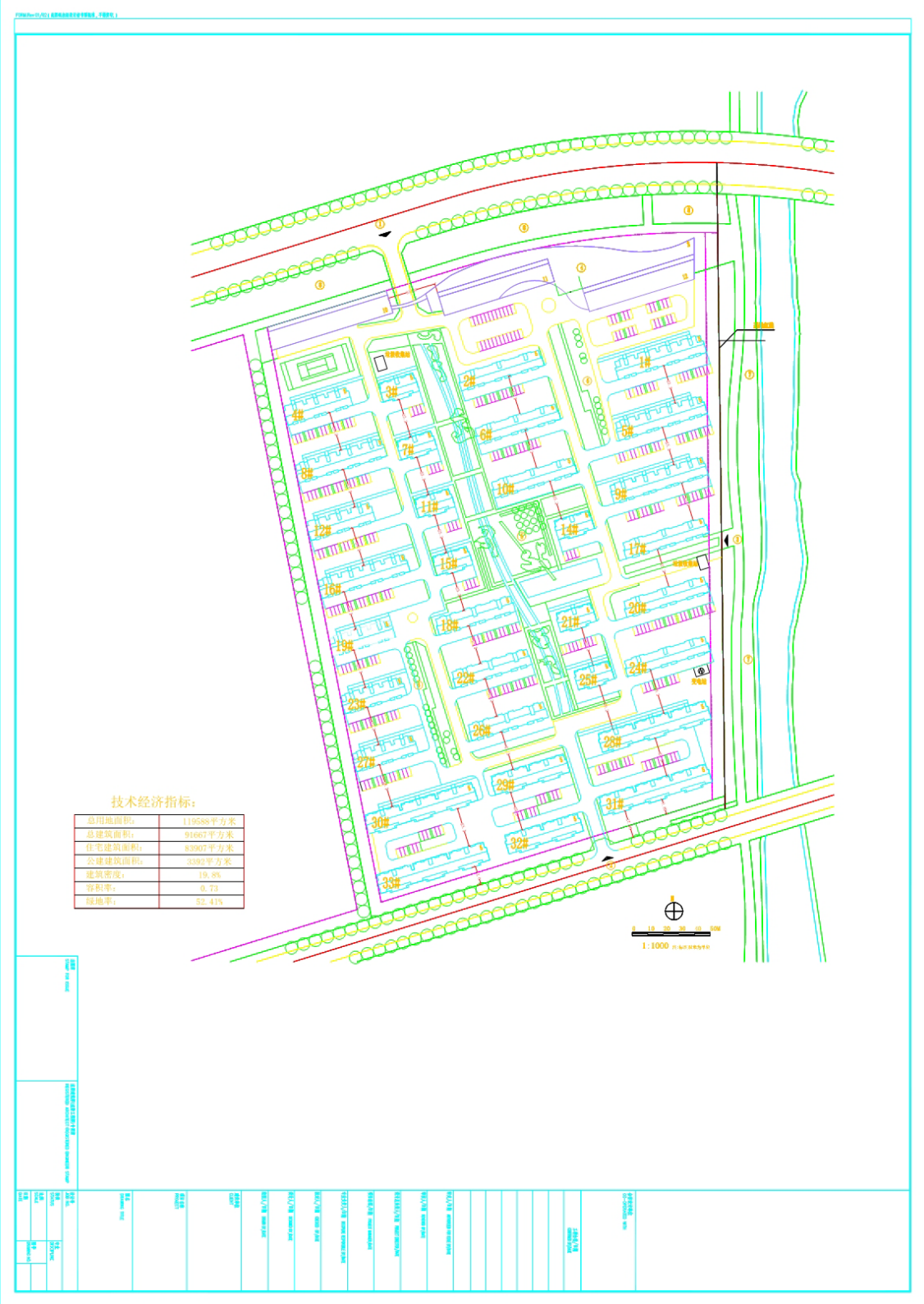

Figure 17. The cross-road actual cell. 


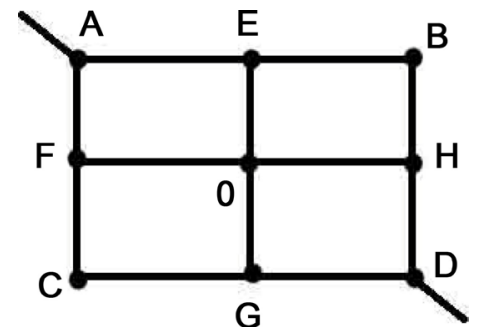

Figure 18. The cross-road map.

Through the analysis of the actual map, we get the following road network simulation (Figure 18).

We set the $A B$ segment for the $n_{1}$ lane, $B D$ segment for the $n_{2}$ lane, $C D$ for the $n_{3}$ lane, $A C$ segment for the $n_{4}$ lane and 1 Lane inside the cell.

After the opening of the district:

According to the principles of the road drivers, the vehicle at $A$ point into the network. $A E$ and $A F$ sections is the same road density so the $A E=\rho$ and $A F=\rho$.

At $E$ point because of the density of the $E B$ segment and the $E O$ section, the density ratio of the two sections is $n_{1}: 1$. Then,

$$
E B=\frac{\rho}{n_{1}+1}, E O=\frac{\rho}{n_{1}+1}
$$

At $F$ point because of the density of the $F C$ segment and the $F O$ section, the density ratio of the two sections is $n_{4}: 1$.Then,

$$
F C=\frac{\rho}{n_{4}+1}, F O=\frac{\rho}{n_{4}+1}
$$

At $O$ point because the density of the $O H$ section and the $O G$ section is the same, the density ratio of the two sections is $1: 1$ and the traffic volume is the sum of the $E O$ and $F O$ sections. Then,

The traffic flow density of $B H$ and $C G$ sections is fully inherited in $E B$ segment and $F C$ segment. Then,

$$
O H=\frac{\left[\frac{\rho}{n_{1}+1}+\frac{\rho}{n_{4}+1}\right]}{2}, O G=\frac{\left[\frac{\rho}{n_{1}+1}+\frac{\rho}{n_{4}+1}\right]}{2}
$$

$H D$ and $G D$ sections of traffic flow density were inherited with $B H$ sections and $O H$ sections with $C G$ sections and $O G$ sections. Then,

$$
G D=\frac{\left\{\rho \times \frac{n_{4}}{n_{4}+1}+\frac{\left[\frac{\rho}{n_{1}+1}+\frac{\rho}{n_{4}+1}\right]}{2}\right\}}{n_{3}}, H D=\frac{\left\{\rho \times \frac{n_{1}}{n_{1}+1}+\frac{\left[\frac{\rho}{n_{1}+1}+\frac{\rho}{n_{4}+1}\right]}{2}\right\}}{n_{2}}
$$

Before the district is not ope, the density of each section:

$$
A B=\rho, B D=\frac{\rho \times n_{1}}{n_{2}}, C D=\frac{\rho \times n_{4}}{n_{3}}, A C=\rho
$$

Final return time: 


$$
\begin{aligned}
& \Delta T=\left[\frac{n_{4}}{n_{4}+n_{1}}\right] \times\left\{\frac{\gamma_{1}}{f\left(D+D_{n}\right)}+\frac{\gamma_{2}}{f\left(D \times \frac{n_{4}}{n_{2}}+D_{i}\right)}-\frac{n_{4}}{n_{4}+1} \times\left[\frac{\gamma_{2}}{f\left(\frac{D}{n_{4}+1}+D_{W}\right)}+\frac{\gamma_{1}}{f\left(D \times \frac{n_{4}}{\left[\left(n_{4}+1\right) \times n_{2}\right]}\right)+D_{i}}\right]\right. \\
& \left.-\frac{1}{n_{4}+1} \times\left[\frac{\gamma_{1}}{f\left[\frac{D}{n_{4}+1}\right]}+\frac{\gamma_{2}}{\left.f\left(\frac{\left[\frac{D}{n_{1}+1}+\frac{D}{n_{4}+1}\right]}{2}\right]\right)}\right]\right\}+\left(\frac{n_{1}}{n_{1}+n_{4}}\right) \times\left\{\frac{\gamma_{1}}{f\left(D+D_{n}\right)}+\frac{\lambda_{2}}{f\left(D \times \frac{n_{1}}{n_{2}}+D_{i}\right)}-\frac{n_{2}}{n_{2}-1}\right. \\
& \left.\times\left[\frac{\gamma_{2}}{f\left(\frac{D}{D_{i}+1}+D_{e}\right)}+\frac{\gamma_{1}}{f\left(\frac{D}{n_{1}+1}\right)+D_{e}}\right]-\frac{1}{n_{2}+1} \times\left[\frac{\lambda_{1}}{f\left(\frac{D}{\frac{n_{1}+1}{2}+\frac{D}{n_{4}+1}}\right)}+\frac{\lambda_{2}}{f\left(\frac{D}{n_{1}+1}\right)}\right]\right\}
\end{aligned}
$$

Through the calculation of the data, we come to the conclusion as Table 2:

We carried out the following inspection of the district traffic through the evaluation system:

We control the number of lines around the area and cell types as well as the traffic flow density adjustment. As a result, we find that the gain time synchronization increases with the increase of density. However, any cell has its upper limit capacity combined with the actual. So it cannot increase the density of traffic flow.

\subsubsection{Park Type District}

This area is within the selected region of a new park area (Figure 19). Garden-style design makes the complex structure of the district. Now, we make an analysis of this problem about more complex. The main road is a one-way street. The main road network structure marked in the map and the area around the road is a two-way street.

We will simplify the interior of this area (Figure 20).

Through the calculation of the data, we come to the conclusion as Table 3. Through the analysis of Park Road District, we found that it is the same as the "cross road". Through the study of the reasons, we find that the park road network structure is almost the same as the "cross road" community after the geometric transformation. This proves the universality of the "cross road" community.

\section{Results}

The State Council of the People's Republic of China issued the Opinions on Further Strengthening the Management of Urban Planning and Construction, The issue of community Open community has become the focus of attention.

Table 2. The benefit time of cross section.

\begin{tabular}{cccccc}
\hline \multicolumn{7}{c}{ Data Sheet } \\
\hline Traffic density & 135 & 142 & 170 & 225 & 325 \\
Cross section of the benefit time & 3.2172 & 3.4178 & 3.9979 & 7.2684 & 26.1015 \\
\hline
\end{tabular}




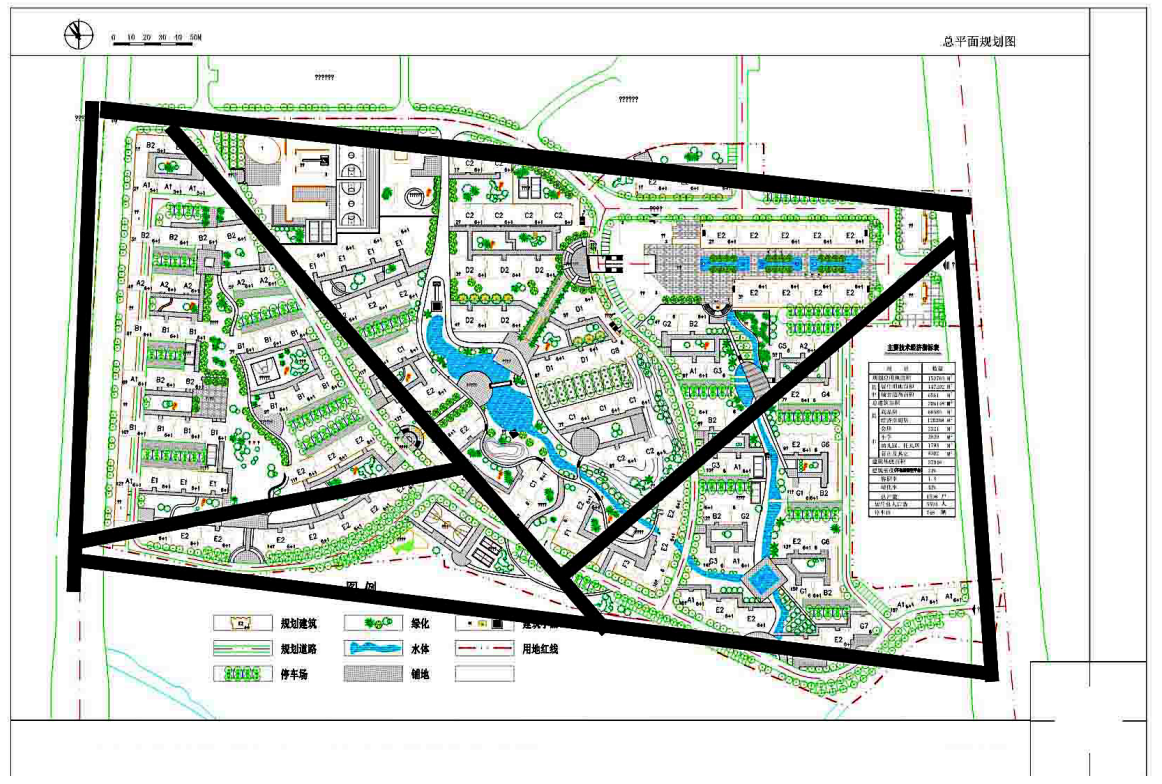

Figure 19. The Park type actual cell.

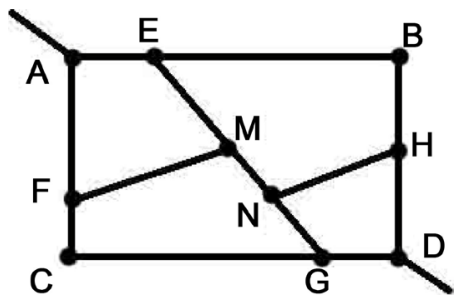

Figure 20. The Park type map.

Table 3. The benefit time of Park-type sections.

\begin{tabular}{cccccc}
\hline \multicolumn{5}{c}{ Data Sheet } & \\
\hline Traffic density & 135 & 142 & 170 & 225 & 325 \\
Park-type sections of the benefit period & 3.2172 & 3.4178 & 3.9979 & 7.2684 & 26.1015
\end{tabular}

Some people disagree with the Open community of the community, taking into account their own security problems. After the opening of the community, with the passage of vehicles increased in the area, people's travel security is not guaranteed, and into the area of mixed personnel, for people's property security is also a great danger. Therefore, in the construction should pay attention to the construction of security measures and internal road construction in parallel to address people's concerns.

After the open area, we can find: with the increasing number of roads within the district, the benefit time gradually increased, but each type of district capacity is different. For the urban planning department, it is possible to increase the road capacity within the area to be built, and the more roads in the area, the stronger the capacity of the area. For the district, has been built for transformation, the internal road construction should be appropriately reduced, not suitable for building too many roads. Because of its internal road structure is fixed, so 
we must fully consider the choice of road width factors.

\section{Conclusions}

Through the establishment of the traffic velocity-density relation model, we can see that the one-way and the T-shaped roads have lower bearing capacity. For the simple structure of the internal road area, it cannot make too much traffic flow through, otherwise it will lead to cell blockage. Therefore, it is necessary to control the traffic volume in the district for traffic management. In the daily traffic peak hours, we must set traffic flow access restrictions to prevent excessive traffic flow.

\section{Acknowledgements}

This research was carried out with support of National Natural Science Foundation of People's Republic of China (project 71261017 and 71661025).

\section{References}

[1] Bressack, M.A., Morton, N.S. and Hortop, J. (1987) Group b Streptococcal Sepsis in the Piglet: Effects of Fluid Therapy on Venous Return, Organ Edema, and Organ Blood Flow. Circulation Research, 61, 659-669. https://doi.org/10.1161/01.RES.61.5.659

[2] Li, J. and Liu, F. (2013) The Planning and Design of Small and Medium Sized Residential Areas in China: Electronic (31).

[3] National Bureau of Statistics of the People's Republic of China (2014) 2011-2013 Focus on Environmental Protection in Key Urban Road Traffic Noise Monitoring Situation. http://www.stats.gov.cn/ztjc/ztsj/hjtjzl/2014/201609/t20160918_1400895.html

[4] Giese, A., Stolwijk, N.A. and Bracht, H. (2000) Double-Hump Diffusion Profiles of Copper and Nickel in Germanium Wafers Yielding Vacancy-Related Information. Applied Physics Letters, 77, 642-644. https://doi.org/10.1063/1.127071

[5] Gower, J.C. and Ross, G.J.S. (1969) Minimum Spanning Trees and Single Linkage Cluster Analysis. Applied Statistics, 18, 54-64. http://www.jstor.org/stable/2346439 https://doi.org/10.2307/2346439

[6] Carvalho, B.M., Gau, C.J., Herman, G.T. and Kong, T.Y. (1999) Algorithms for Fuzzy Segmentation. International Conference on Advances in Pattern Recognition, Plymouth, 23-25 November 1998, 154-163.

http://link.springer.com/chapter/10.1007/978-1-4471-0833-7_16 https://doi.org/10.1007/978-1-4471-0833-7_16

[7] Manen, S., Guillaumin, M. and Gool, L.V. (2013) Prime Object Proposals with Randomized Prim's Algorithm. IEEE International Conference on Computer Vision, Sydney, 1-8 December 2013, 2536-2543.

http://www.cv-foundation.org/openaccess/content_iccv_2013/papers/Manen_Prim e_Object_Proposals_2013_ICCV_paper.pdf https://doi.org/10.1109/iccv.2013.315

[8] Abdel-Aziz, Y.I. (1971) Direct Linear Transformation from Comparator Coordinates in Close-Range Photogrammetry. ASP Symposium on Close-Range Photogrammetry in Illinois, Champaign, 26-29 January 1971, 1-18.

[9] Mikhail, E.M., Bethel, J.S. and McGlone, J.C. (2001) Introduction to Modern Pho- 
togrammetry. Wiley, New York.

[10] Abdel-Aziz, Y.I., Karara, H.M. and Hauck, M. (2015) Direct Linear Transformation from Comparator Coordinates into Object Space Coordinates in Close-Range Photogrammetry. Photogrammetric Engineering \& Remote Sensing, 81, 103-107. https://doi.org/10.14358/PERS.81.2.103

[11] Glantz, S.A. and Slinker, B.K. (1990) Primer of Applied Regression and Analysis of Variance. Health Professions Division, McGraw-Hill, New York.

[12] Fox, J. (1997) Applied Regression Analysis, Linear Models, and Related Methods. Sage Publications, Inc., Thousand Oaks.

[13] Montgomery, D.C., Peck, E.A. and Vining, G.G. (2015) Introduction to Linear Regression Analysis. John Wiley \& Sons, Hoboken.

[14] Qiu, C., Zuo, X., Wang, C. and Wu, J. (2010) A BP Neural Network Based Information Fusion Method for Urban Traffic Speed Estimation. Engineering Sciences, 8, 77-83.

[15] Chan, K.Y., Dillon, T.S., Singh, J. and Chang, E. (2012) Neural-Network-Based Models for Short-Term Traffic Flow Forecasting Using a Hybrid Exponential Smoothing and Levenberg-Marquardt Algorithm. IEEE Transactions on Intelligent Transportation Systems, 13, 644-654. https://doi.org/10.1109/TITS.2011.2174051

[16] Barba, L. (2014) Forecast of Traffic Accidents Based on Components Extraction and an Autoregressive Neural Network with Levenberg-Marquardt. In: Prasath, R., O'Reilly, P. and Kathirvalavakumar, T., Eds., Mining Intelligence and Knowledge Exploration, Springer International Publishing, New York, 82-90.

https://doi.org/10.1007/978-3-319-13817-6_9

\section{Scientific Research Publishing}

Submit or recommend next manuscript to SCIRP and we will provide best service for you:

Accepting pre-submission inquiries through Email, Facebook, LinkedIn, Twitter, etc. A wide selection of journals (inclusive of 9 subjects, more than 200 journals)

Providing 24-hour high-quality service

User-friendly online submission system

Fair and swift peer-review system

Efficient typesetting and proofreading procedure

Display of the result of downloads and visits, as well as the number of cited articles

Maximum dissemination of your research work

Submit your manuscript at: http://papersubmission.scirp.org/

Or contact jamp@scirp.org 